\title{
Evaluación del control interno basado en el modelo COSO 2013 para mejorar la gestión logística de la empresa REDJOS S.A.C., año 2018
}

Evaluation of internal control based on the COSO 2013 model to improve the logistics management of the company REDJOS S.A.C., year 2018

\section{Daniel Tejada}

danotejada@gmail.com

Código ORCID: 0000-0002-7970-3598

Universidad Peruana Unión - Perú

\author{
Giner Guadalupe \\ ginerobedg@gmail.com \\ Código ORCID: 0000-0002-3073-1600 \\ Universidad Peruana Unión - Perú
}

Artículo recibido abril 2019 / Arbitrado en mayo 2019 / Publicado en julio 2019

RESUMEN La apertura de nuevos mercados y las exigencias de los clientes en calidad de productos, servicios y precios competitivos, señala el camino por donde las empresas deben trazar sus objetivos para sobrevivir a la exigencia de un mercado competitivo, por ello es de suma importancia que toda organización tenga garantizado una buena gestión en un control interno efectivo y eficiente que genere una seguridad razonable para la consecución de los objetivos de la entidad. Es en este sentido que el objetivo general de la presente investigación fue evaluar el control interno basado en el modelo COSO 2013 para mejorar la gestión logística de la empresa Redjos S.A.C, 2018. El tipo de estudio fue descriptiva inductiva con un diseño no experimental transversal, no experimental debido a que se observó el fenómeno en su forma habitual para posteriormente analizarlos y transversal porque se recolectó los datos en un solo momento. Para el estudio realizado se utilizó el cuestionario de control interno basado en el modelo COSO 2013 en base a los 17 principios para la población de la dirección de la empresa y una ficha de observación para identificar las deficiencias de los procesos logísticos. En concordancia con los resultados se determinó que la empresa viene aplicando con eficacia el control interno en un $13.22 \%$, asimismo un $47.67 \%$ parcialmente eficaz y no eficaz en un $39.11 \%$, asimismo respecto a los procesos logísticos se identificó una serie de riesgos y actividades de control deficientes, por ende, resulta necesario la aplicación de un sistema de control interno eficiente y efectivo para mejorar la gestión logística.

Palabras clave: Control interno; eficiente; efectivo; mercados competitivos; objetivos; gestión logística; riesgos; actividades de control; procesos logísticos

ABSTRACT and competitive prices, points the way through which companies must draw their objectives to survive the demand of a competitive market, therefore it is of the utmost importance that all The organization has guaranteed good management in an effective and efficient internal control that generates reasonable assurance for the achievement of the entity's objectives. It is in this sense that the general objective of this research was to evaluate the internal control based on the COSO 2013 model to improve the logistics management of the company Redjos SAC, 2018. The type of study was descriptive inductive with a non-experimental cross-sectional design, non-experimental because the phenomenon occurred in its usual way to later analyze them and cross-sectional because the data was collected in a single moment. For the study carried out, the internal control questionnaire based on the COSO 2013 model was used, based on the 17 principles for the population of the company's management and an observation form to identify the deficiencies of the logistics processes. In accordance with the results, it was determined that the company has been effectively applying internal control in $13.22 \%$, also in $47.67 \%$ partially effective and not effective in $39.11 \%$, also with regard to logistics processes, a series of risks and activities were identified deficient control systems, therefore, it is necessary to apply an efficient and effective internal control system to improve logistics management.

Keywords: Internal control; efficient; effective; competitive markets; objectives; logistics management; risks; control activities; logistics processes 


\section{INTRODUCCIÓN}

En el universo empresarial, las diferentes organizaciones se encuentran en una lucha por la supervivencia y crecimiento económico, con el fin de llegar a ser sostenibles y mantenerse en un mercado competitivo. Para ello, dependen sostenerse de las herramientas del control interno y la gestión de logística.

El control interno desde hace mucho tiempo ha sido reconocido como fundamental e indispensable en la actividad empresarial y en la práctica de la auditoría, en tal sentido en la última década los controles internos han tomado una orientación dirigida de manera prioritaria a fomentar la eficiencia, reduciendo el riesgo en la consecución de los objetivos, ayudando a asegurar la confiabilidad de la información financiera, la gestión de los recursos, y a cumplir las leyes, reglamentos y cláusulas contractuales aplicables (Rivas, 2011).

Desde este contexto la aplicación de un adecuado control interno es importante dentro de este modelo de organizaciones para lograr optimizar los recursos y fortalecer el desempeño de las empresas tanto en la parte operativa como en lo administrativo, consiguiendo multiplicar las utilidades $y$ aprovechando la oportunidad externa de nuevas inversiones (Álvarez y Durán citado por Ubillús, Tamayo, y Zambrano, 2016).

Por otro parte Young (2013) señala que las grandes empresas incorporan en sus procesos un conjunto de medidas preventivas (control interno) que contribuyen a minimizar los riesgos. Al respecto Servin (2018) menciona que las pequeñas y medianas empresas no cuentan con un control interno razón por el cual trabajan empíricamente dando soluciones a los problemas conforme se presenten, debilitando de esta manera las decisiones de la gerencia respecto al cumplimiento de los objetivos empresariales.

Entre tanto la logística juega un papel importante cuando se requiere obtener una ventaja competitiva respecto a los demás competidores ya que conlleva a las empresas a ser más eficientes y productivos en los diferentes procesos de abastecimiento (García, 2010). Razón por el cual "una logística eficiente y accesible a todos constituye un elemento clave para que las empresas de determinado país y en particular sus PYMES, puedan competir con éxito en este nuevo marco global" (Kirby y Brosa, 2011).

Actualmente la empresa Redjos S.A.C, ha logrado posicionarse en el mercado nacional, manteniendo contratos comerciales con grandes empresas corporativas tales como, Cineplex S.A, Villa Natura Perú S.A.C, Bembos S.A.C, Hipermercados Tottus S.A, Snack América Latina S.R.L, Uvk Multicines S.A, Grupo Cirsa, Perufarma S.A y diversas distribuidoras que comercializan snacks a nivel nacional, esto conllevó a que la entidad se tenga que adecuar aceleradamente a los requerimientos de estas empresas, generando deficiencias en las actividades logísticas de producción, almacenamiento y distribución, repercutiendo esto en la falta de planificación en el aprovisionamiento de materiales, flexibilidad en la programación de la producción y retraso en la atención de productos, cabe señalar también que no existe un sistema de almacenamiento adecuado debido a la ausencia de herramientas de control de inventarios, que registre $y$ actualice las existencias.

Por otro lado, la falta de coordinación entre los departamentos de ventas, producción y compras impide que la información de los productos almacenados sea confiable, a su vez 
Daniel Tejada y Giner Guadalupe

esto afecta el control efectivo sobre el tiempo de vida útil de los productos. Existe también la falta de un seguimiento sistemático en la entrega de pedidos, generando todo ello pérdidas económicas para la empresa, por lo tanto, surge la necesidad de evaluar el control interno para mejorar la gestión logística a partir del cual la empresa podrá obtener un producto final a un menor costo y tiempo, representando una ventaja competitiva importante frente a sus demás competidores.

La presente investigación surge a partir de conocer la problemática que vienen afrontando a través de los años las pequeñas y medianas empresas que no emplean el control interno dentro de su estructura organizacional. Por lo cual la empresa Redjos SAC, no es ajena a esta problemática, razón por la que se ha visto afectado financieramente, como en su desempeño empresarial competitivo y eficiente en el mercado. En este contexto de pertinencia la importancia de la presente investigación es proponer una herramienta de evaluación del control interno que contribuya a mejorar la gestión logística de la empresa, objeto de estudio. Por consiguiente, la presente investigación evaluará la gestión logística de la empresa y las actividades de producción, almacenamiento y distribución haciendo uso de instrumentos de control interno según el modelo COSO 2013.

\section{MATERIALES Y METODO}

Es una investigación descriptiva inductiva que no considera hipótesis porque se pretende conocer la situación actual del control interno para luego analizarlas, describir e interpretar los fenómenos que ocurren en la organización. Al respecto Hernández, Fernández, \& Baptista (2014) señalan que "en los estudios descriptivos se busca especificar las propiedades, las características y los perfiles de personas, grupos, comunidades, procesos, objetos o cualquier otro fenómeno que se someta a un análisis".

Por otro lado, es de un diseño no experimental transversal, experimental puesto que se mira el fenómeno en su forma habitual para posteriormente analizarlos, y transversal porque recolectan datos en un solo momento, en un tiempo único. Su finalidad es describir variables, y analizar su incidencia e interrelación en un solo momento Hernández, Fernández, \& Baptista (2014).

En cuanto a las técnicas de recolección de datos, se utilizaron la ficha de observación para conocer las deficiencias en el área de logística de la empresa Redjos S.A.C, y el cuestionario, el cual se realizó con la finalidad de conocer la situación actual del control interno para mejorar la gestión logística de la empresa Redjos S.A.C, dicho instrumento se elaboró en base a los 17 principios del modelo COSO 2013 y se aplicará a al gerente general, administrador y contador.

Los datos obtenidos en la encuesta se analizarán utilizando la herramienta hoja de cálculo Excel y se elaborarán indicadores estadísticos del momento actual. Asimismo, se elaborarán flujogramas del proceso logísticos para luego analizarlas en la matriz de evaluación del control interno.

\section{RESULTADOS Y DISCUSIÓN}

El presente instrumento de investigación es propio del marco integrado COSO 2013, estructurado con base en los 17 principios debidamente validados y fiables, que sirven como guía para diseñar, implementar y evaluar el control interno en una organización. 


\section{Información de los responsables del control interno de la empresa}

Tabla 1. Directivos y profesionales

\begin{tabular}{lc}
\hline & Directivos y Profesionales \\
\hline Gerente General & Directivo \\
Administrador & Profesional \\
Contador & Profesional \\
\hline
\end{tabular}

Fuente: Los investigadores (2020)

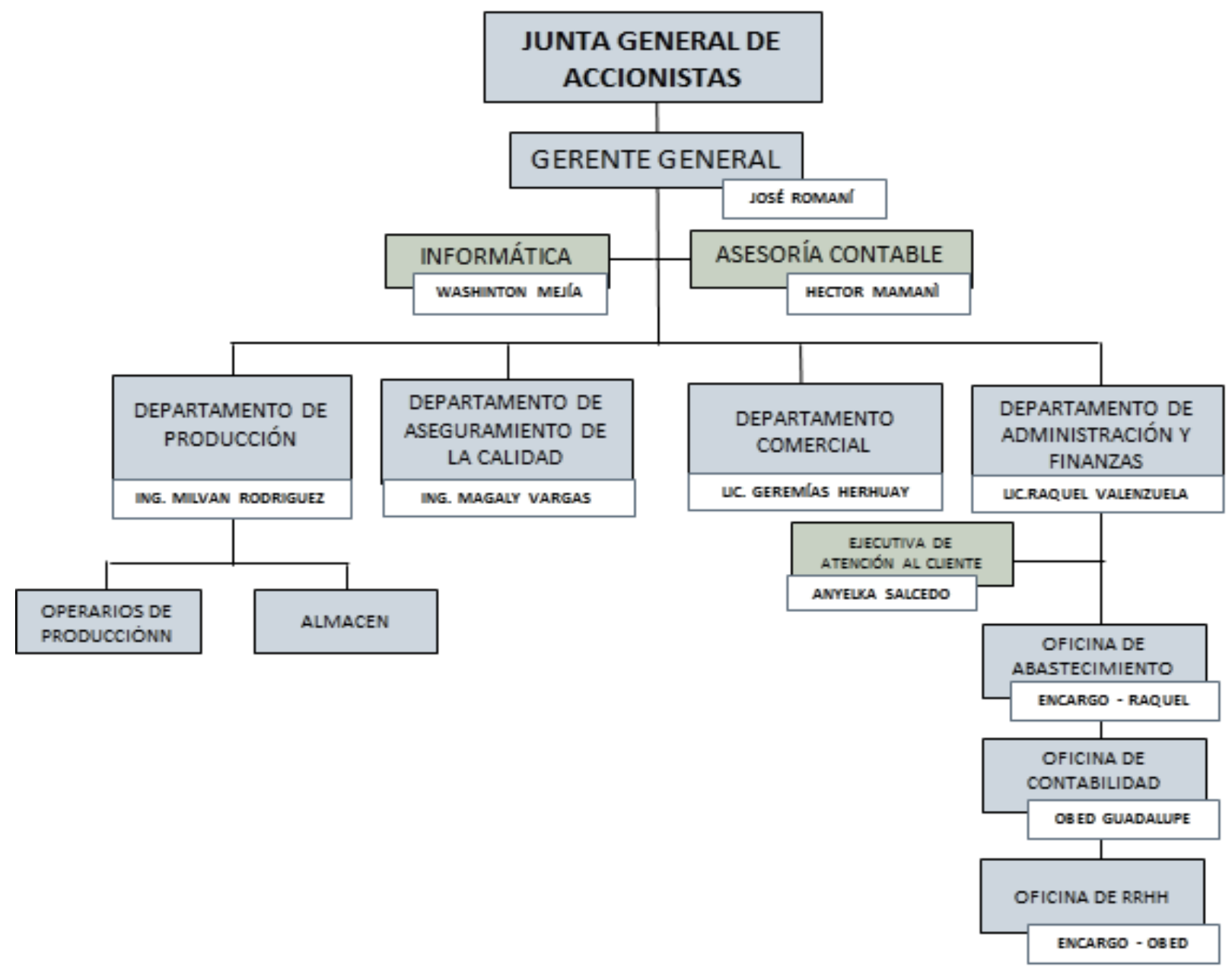

Figura 1 Organigrama actual Redjos S.A.C. Fuente: Redjos S.A.C. 
Daniel Tejada y Giner Guadalupe

\section{Descripción de la situación actual del Control Interno de la empresa}

La globalización y la apertura de nuevos mercados ha conllevado a que la empresa Redjos S.A.C se vea en la necesidad de adaptarse a cambios tecnológicos, de entorno y competencia de manera empírica, sin embargo es necesario que se apliquen controles eficientes y efectivos que permitan que los objetivos se puedan alcanzar de manera confiable, por lo cual es de suma importancia que se considere un modelo de control interno que sirva como guía para la evaluación e implementación de un sistema de control interno.

\section{Entorno de Control.}

Tabla 2. Descripción de la situación actual del Entorno de Control

\section{Principios Modelo COSo \\ Empresa Redjos S.A.C}

Este principio hace referencia a una entidad desarrolla y utiliza un código formal de códigos de conducta y otras políticas para comunicar normas de conducta.

Hace referencia a una entidad donde existe independencia entre el que supervisa el control interno de aquel que lo desarrolla e implementa.

3) Establecer la estructura, la autoridad y responsabilidad

4) Demostrar compromiso con las competencias.
Hace referencia a una entidad en donde el Gerente General mediante la asesoría externa, establece estructuras, líneas de reporte y responsabilidades apropiadas para la consecución de los objetivos.

Este principio se refiere a una entidad que demuestra compromiso por atraer, desarrollar y retener individuos competentes con sus objetivos.
La empresa solo transmite mensajes de buena conducta mediante el actuar del personal de dirección y en algunas reuniones sociales que se convocan.

Actualmente la empresa no cuenta con un comité de control interno establecido, por lo que la supervisión de los controles se realiza de forma empírica.

La empresa cuenta con un organigrama, sin embargo, este carece de líneas de reporte y asignación de responsabilidades.

La empresa no realiza evaluaciones de desempeño de trabajo, el personal que ingresa a laborar en muchas veces es por recomendación, por otra parte, solo se realiza capacitaciones para el personal de producción, pero no es continuo. 


\section{Principios}

5) Aplicar la rendición de cuentas.

\section{Modelo COSO}

Este principio hace referencia a una entidad que cuenta con personal responsable del control interno para la consecución de los objetivos.

\section{Empresa Redjos S.A.C}

No se cuenta con responsables que supervisen el buen manejo del control interno, sin embargo, consideramos que es importante que asigne responsabilidades a los jefes de área con la finalidad que se tomen acciones correctivas inmediatas cuando consideren que los objetivos se desvían.

Fuente: Tejada, Guadalupe 2019.

\section{Evaluación de Riesgos.}

Tabla 3. Descripción actual de la evaluación de riesgos

\begin{tabular}{ccc}
\hline Principios & Modelo coso & Empresa Redjos S.A.C \\
\hline
\end{tabular}

6) Especificar objetivos adecuados

7) Identificar y analizar riesgos

8) Evaluar el riesgo de fraude
Este principio hace referencia a una entidad que especifica sus objetivos con suficiente claridad para permitir la identificación y evaluación de riesgos relacionados a los objetivos.
Este principio hace referencia a una entidad que identifica riesgos que afectan el logro de sus objetivos y que analiza cómo deben ser gestionados.

Este principio hace referencia a una entidad que considera la posibilidad de fraude en la evaluación de riesgos contra el logro de fraude.
La dirección de la empresa estable solo objetivos comerciales mediante la comunicación por correo electrónico, más consideramos importante que todas las áreas de la empresa participen en la elaboración de un plan estratégico que sirva como referencia para identificar $y$ evaluar los riesgos que puedan afectar los objetivos.

Parcialmente, la empresa realiza la gestión de riesgos de manera empírica, ya que no cuenta con un plan estratégico.

No, la dirección de la empresa no considera un fraude como un factor que pueda afectar a la empresa, sin embargo, consideramos importante que toda entidad debe de contar con procedimientos que ayuden a detectar todo tipo de fraude. 
Daniel Tejada y Giner Guadalupe

\section{Principios}

9) Identificar y analizar cambios significativos

\section{Modelo COSO}

Este principio hace referencia a una entidad que identifica y evalúa los cambios que podrían afectar impactar significativamente al sistema de control interno.

\section{Empresa Redjos S.A.C}

No, la dirección de la empresa mediante la gestión de su Gerente General evalúa los cambios en el entorno externo que pudieran afectar los objetivos de la entidad. Por ello consideramos importante que se documente los procedimientos para la evaluación de los cambios en el entorno externo.

Fuente: Tejada, Guadalupe 2019.

\section{Actividades de Control.}

Tabla 4. Descripción actual de las actividades de control

\section{Principios}

10) Seleccionar y desarrollar actividades de control.

11) Seleccionar y desarrollar controles generales sobre tecnología.

\section{Modelo coso}

Empresa Redjos S.A.C

Este principio hace referencia a una entidad que define $y$ desarrolla actividades de control que contribuyen a la mitigación de riesgos.

Este principio hace referencia a una entidad que ha definido y desarrollado actividades de control para la tecnología de la información con el fin de apoyar la consecución de los objetivos.
Parcialmente, la empresa realiza actividades control dirigidas por sus jefes de área, sin embargo, carece de formalidad $y$ documentación debido a que solo mitigan el riesgo en el momento que se presenta. Por ello es importante establecer controles que estén orientados al cumplimiento de los objetivos operacionales, de confiabilidad de la información financiera y el cumplimiento de leyes.

$\mathrm{Si}$, la empresa tiene implementado un sistema de gestión ERP, que provee de información financiera y no financiera para el cumplimiento de los objetivos, sin embargo, es necesario que se implementen procesos eficientes que contribuyan en mitigar los riesgos. 


\section{Principios}

12) Implementación a través de políticas y procedimientos.

\section{Modelo Coso}

Este principio hace referencia a una entidad que despliega las actividades de control a través de políticas que establecen las líneas generales del control interno.
Empresa Redjos S.A.C

Parcialmente, el gerente general es quien evalúa el desempeño de los procesos, actividades y tareas que se realizan en la empresa, asimismo imparte instrucciones mediante correo electrónico, sin embargo, consideramos necesario que se implemente un registro de los indicadores de desempeño para los procedimientos, actividades y tareas, por otro lado, también es importante que se establezcan políticas y procedimientos de responsabilidad.

Fuente: Tejada, Guadalupe 2019.

Información y comunicación.

Tabla 5. Descripción de la situación actual de la Información y comunicación

Principio

Modelo COSO
Empresa Redjos S.A.C
13) Utilizar información pertinente.
Este principio hace referencia a una entidad que obtiene, genera y emplea información relevante y de calidad para apoyar el funcionamiento del control interno.
Parcialmente, la dirección de la empresa considera relevante la información procesada por el sistema de gestión, para la toma de decisiones operativas. Sin embargo, para que la información sea de calidad y útil para la toma de decisiones, el modelo COSO propone que para comprobar la calidad de información se analicen las siguientes preguntas:

¿Contiene toda la información necesaria?

¿Se facilita en el tiempo adecuado? ¿Es la más reciente disponible? ¿Los datos son correctos? ¿Puede ser obtenida fácilmente por las personas adecuadas? 
Daniel Tejada y Giner Guadalupe

Principio

14) Utilizar información pertinente.

\section{Modelo Coso}

Este principio hace referencia a una entidad que obtiene, genera y emplea información relevante y de calidad para apoyar el funcionamiento del control interno.
15) Comunicación interna.

16) Comunicación externa.
Este principio hace referencia a una entidad que comunica la información internamente, incluyendo los objetivos y responsabilidades del control interno necesarios para apoyar el funcionamiento del control interno.

Este principio hace referencia a una entidad que comunica a terceras partes externas lo referente a los asuntos que afectan el funcionamiento del control interno.
Parcialmente, la dirección de la empresa considera relevante la información procesada por el sistema de gestión, para la toma de decisiones operativas. Sin embargo, para que la información sea de calidad y útil para la toma de decisiones, el modelo COSO propone que para comprobar la calidad de información se analicen las siguientes preguntas:

¿Contiene toda la información necesaria?

¿Se facilita en el tiempo adecuado?

¿Es la más reciente disponible? ¿Los datos son correctos? ¿Puede ser obtenida fácilmente por las personas adecuadas?

No, la empresa no cuenta con políticas y procedimientos que suministren de información al personal para el cumplimiento de sus funciones, solo se cuenta con un periódico mural. Por ello consideramos importante contar con canales de comunicación interna que favorezcan la comunicación de fallas del control interno.

Parcialmente, la dirección de la empresa guarda una buena relación con sus proveedores y clientes, sin embargo, no se cuenta con canales de comunicación establecidos, por lo que resulta importante documentar dichos procedimientos, de esta forma se podrá contar con una comunicación externa confiable y segura.

Fuente: Tejada, Guadalupe 2019. 


\section{Actividades de Monitoreo.}

Tabla 6. Descripción actual de las actividades de monitoreo

Principios

17) Realizar evaluaciones continuas $\mathrm{y} / \mathrm{o}$ separadas.

Modelo COSO

Este principio hace referencia a una entidad que selecciona, desarrolla y ejecuta evaluaciones continuas $\mathrm{y} / \mathrm{o}$ periódicas para comprobar si los componentes de control interno están presentes y en operación.

18) Evaluar y comunicar deficiencias.
Este principio hace referencia a una entidad que comunica las deficiencias del control interno de manera oportuna a las partes responsables de tomar acciones correctivas.
Empresa Redjos S.A.C
No, la dirección no realiza un monitoreo de los controles internos, solo el gerente general es quien de manera discrecional evalúa los controles aplicados. Por ello es pertinente que se realice evaluaciones continúas articuladas con los procesos más importantes de la entidad.

Parcialmente, los jefes de área cuando consideran necesario comunican las deficiencias de algún control al gerente. Pero no existe un procedimiento establecido para realizar el seguimiento de la acción correctiva.

Fuente: Tejada, Guadalupe 2019.

\section{Flujograma actual del área logística de la empresa}

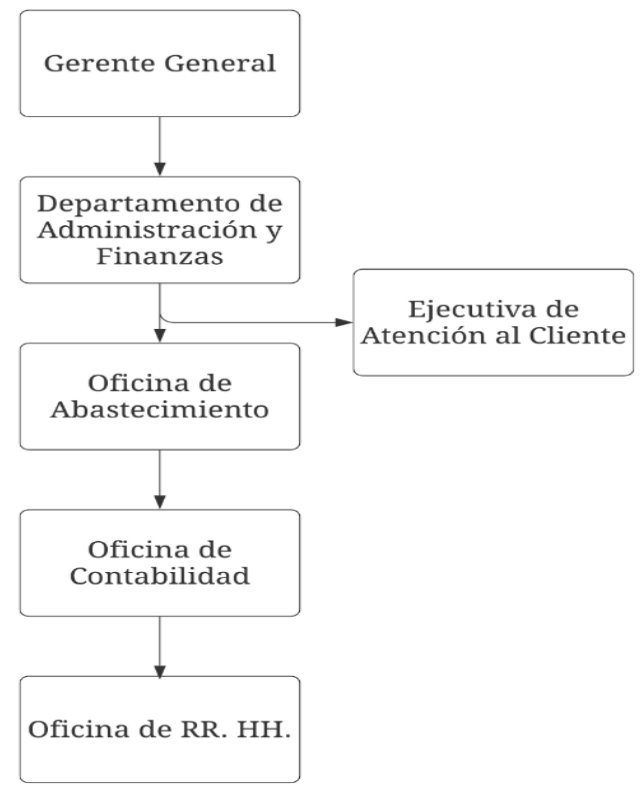

Figura 2. Organigrama actual del área logística. Fuente: Rejos S.A.C 
Daniel Tejada y Giner Guadalupe

Como se observa en la (figura 2) la empresa Redjos S.A.C no cuenta con una estructura orgánica establecido para el área logística por lo que consideramos que la empresa no le da tanta importancia a este departamento, por otro lado, podemos mencionar que la estructura orgánica actual tiene una posición cuyo peso recae más en el departamento comercial ya que dirección prioriza más la gestión comercial, como son las ventas y el captar más clientes.

Dada la importancia de la evaluación del control interno para mejorar la gestión logística, se aplicó una encuesta basada en el modelo COSO 2013, para luego tabular y presentar la información por cada componente.

Tabla 7. Cuestionario de Entorno de Control del modelo COSO, y Redjos S.A.C

\begin{tabular}{ccc}
\hline Opción de respuesta & Puntuación & Porcentaje \\
\hline Sí & 2 & $13 \%$ \\
Sí, parcialmente & 7 & $47 \%$ \\
No & 6 & $40 \%$ \\
Total & 15 & $100 \%$ \\
\hline
\end{tabular}

Fuente: Tejada, Guadalupe 2019.

\section{Análisis}

Los resultados reflejan que en un $47 \%$ el entorno de control es parcialmente eficaz en cuanto a la aplicación de normas, procesos y estructuras que rigen dentro de ella, sin embargo, un $40 \%$ es no eficaz, esto debido a que la entidad carece de formalidad, documentación y compromiso de todo el personal con los objetivos que persigue la empresa, finalmente un $13 \%$ del entorno de control es eficaz, esto debido a que la dirección en base a la experiencia ha implementado una filosofía de gestión y administración estratégica que ha llevado a que la entidad se adapte fácilmente a las exigencias del mercado.

Tabla 8. Cuestionario de Evaluación de Riesgos según COSO, y Redjos S.A.C.

\begin{tabular}{ccc}
\hline Opción de respuesta & Puntuación & Porcentaje \\
\hline Sí & 1 & $8.33 \%$ \\
Sí, parcialmente & 1 & $8.33 \%$ \\
No & 10 & $83.33 \%$ \\
Total & 12 & $100 \%$ \\
\hline
\end{tabular}

Fuente: Tejada, Guadalupe 2019. 


\section{Análisis}

Los resultados muestran que un $83.33 \%$ de la evaluación de riesgos es no eficaz, esto debido a que la entidad no cuenta con un plan estratégico que lo conlleve a identificar y evaluar los riesgos que pueden afectar los objetivos, por otra parte, un $8.33 \%$ parcialmente si es eficaz, la dirección de la empresa mediante la intervención de los jefes de área gestiona los riesgos de manera empírica conforme se presenten en las actividades, y finalmente un $8.33 \%$ de la evaluación de riesgo es eficaz debido a que el gerente general siempre ha dado respuesta a los riesgos que puedan afectar los objetivos de la entidad.

Tabla 9. Cuestionario de Actividades de Control según COSO, y Redjos S.A.C.

\begin{tabular}{ccc}
\hline Opción de respuesta & Puntuación & Porcentaje \\
\hline Sí & 3 & $33.33 \%$ \\
Sí, parcialmente & 6 & $67.67 \%$ \\
No & 0 & $0.00 \%$ \\
Total & 9 & $100 \%$ \\
\hline
\end{tabular}

Fuente: Tejada, Guadalupe 2019.

\section{Análisis}

Los resultados muestran que el $66.67 \%$ parcialmente si es eficaz, debido a que la empresa aplica actividades de control, pero estas no se encuentran debidamente documentadas y formalizadas, por otro lado, un $33.33 \%$ si es eficaz, debido a que la empresa tiene actividades de control establecidos por la gerencia que se cumplen eficientemente y lo cual han conllevado a mitigar los riesgos que pueden afectar los objetivos.

Tabla 10. Cuestionario de Información y Comunicación según COSO, y Redjos S.A.C.

\begin{tabular}{ccc}
\hline Opción de respuesta & Puntuación & Porcentaje \\
\hline Sí & 1 & $11.11 \%$ \\
Sí, parcialmente & 6 & $66.67 \%$ \\
No & 2 & $22.22 \%$ \\
Total & 9 & $100 \%$ \\
\hline
\end{tabular}

Fuente: Tejada, Guadalupe 2019. 
Daniel Tejada y Giner Guadalupe

\section{Análisis}

Los resultados muestran que $66.67 \%$ parcialmente la información y comunicación en la entidad es eficaz, debido a que la empresa genera, identifica y procesa información ya se financiera o no financiera para la toma de decisiones, sin embargo carecen de procedimientos eficientes para que la información sea confiable, por otro lado un $22.22 \%$ es no eficaz, finalmente $11.11 \%$ es eficaz esto debido a que la entidad cuenta con un sistema de gestión ERP que provee de información para la toma de decisiones financieras.

Tabla 11. Cuestionario de Actividades de Monitoreo y supervisión según COSO, y Redjos S.A.C.

\begin{tabular}{ccc}
\hline Opción de respuesta & Puntuación & Porcentaje \\
\hline Sí & 0 & $0.00 \%$ \\
Sí, parcialmente & 3 & $50.00 \%$ \\
No & 3 & $50.00 \%$ \\
Total & 6 & $100 \%$ \\
\hline
\end{tabular}

Fuente: Tejada, Guadalupe 2019.

\section{Análisis}

Los resultados muestran que en un $50 \%$ las actividades de monitoreo es parcialmente eficaz, y un $50 \%$ es no eficaz, en relación con lo que parcialmente se viene aplicando, la dirección de la entidad, de la mano con sus jefes de área realiza un diagnóstico de los controles que se vienen aplicando, pero no siguen una línea base para mejorar los procesos, por otro lado, también se realiza un adecuado seguimiento a las acciones correctivas sin embargo no se cuenta con procedimientos adecuados.

Tabla 12. Resultado de la situación actual del control interno en la empresa Redjos S.A.C.

\begin{tabular}{cccccccc}
\hline $\begin{array}{c}\text { Resultado } \\
\text { Final } \\
\text { (Baremo) }\end{array}$ & $\begin{array}{c}\text { Entorno de Evaluación } \\
\text { control }\end{array}$ & $\begin{array}{c}\text { Actividades } \\
\text { de riesgo }\end{array}$ & $\begin{array}{c}\text { Información y } \\
\text { de control }\end{array}$ & $\begin{array}{c}\text { Actividades } \\
\text { Comunicación }\end{array}$ & $\begin{array}{c}\text { de } \\
\text { Monitoreo }\end{array}$ & Total & $\begin{array}{c}\text { Porcentaje } \\
\text { Final }\end{array}$ \\
\hline Sí & $13.33 \%$ & $8.33 \%$ & $33.33 \%$ & $11.11 \%$ & $0.00 \%$ & $\mathbf{6 6 \%}$ & $13.22 \%$ \\
Parcial & $46.67 \%$ & $8.33 \%$ & $66.67 \%$ & $66.67 \%$ & $50.00 \%$ & $\mathbf{2 3 8 \%}$ & $47.67 \%$ \\
No & $40.00 \%$ & $83.33 \%$ & $0.00 \%$ & $22.22 \%$ & $50.00 \%$ & $\mathbf{1 9 6 \%}$ & $39.11 \%$ \\
Total & $\mathbf{1 0 0 \%}$ & $\mathbf{1 0 0 \%}$ & $\mathbf{1 0 0 \%}$ & $\mathbf{1 0 0 \%}$ & $\mathbf{1 0 0 \%}$ & $\mathbf{5 0 0 \%}$ & $100 \%$ \\
\hline
\end{tabular}

Fuente: Tejada, Guadalupe 2019. 
En correspondencia a los resultados obtenidos, se determinó que la empresa Redjos S.A.C. viene aplicando con eficacia el control interno en un $13.22 \%$, asimismo un $47.67 \%$ parcialmente eficaz y no eficaz en un $39.11 \%$, dicha evaluación evidencia que la entidad viene aplicando un control interno de manera empírica lo cual le ha sido útil hasta la actualidad, sin embargo ante el continuo crecimiento en volumen de activos, producción, ventas y de personal, es necesario que se preste mayor atención al control interno ya que es una herramienta que le permitirá a la entidad adaptarse a los constantes cambios de un mercado competitivo y además de alcanzar los objetivos de manera confiable y segura.

\section{Descripción del proceso actual de aprovisionamiento}

- Recepción de requerimiento de compra: el administrador recibe los requerimientos de compras de todas las áreas, mediante correo electrónico, mensajes de texto de wasap u/o de manera verbal.

- Evaluar requerimiento y verificar stock: el administrador solicita el stock actualizado de las existencias para su verificación, luego procede a asignar al proveedor, para finalmente emitir la orden de compra. Por otro lado, si se verifica que no existe necesidad de compra, se rechaza requerimiento y se notifica al departamento que corresponde.

- Despacho de mercadería: el proveedor recibe la notificación de orden de compra y confirma fecha de atención de acuerdo con las especificaciones.

- Recepción de mercadería: el asistente de control de calidad realiza la recepción de mercadería, antes verifica si los productos a recibir cumplen con los requisitos establecidos en la orden de compra y también evalúa la calidad de la mercadería, si no cumple con los requisitos se rechaza de lo contrario se ingresa a almacén. Por otra parte, el asistente de control de calidad remite los documentos que intervienen en el proceso de despacho al administrador.

- Recepción de documentación: el administrador recibe los documentos que intervienen en el proceso y realiza el registro de la factura en el sistema ERP para su respectiva cancelación.

\section{Descripción del proceso actual de producción}

- Emisión de órdenes de producción: el jefe de producción genera la orden de producción y hojas de trabajo para luego entregar al personal responsable de la producción del día.

- Recepción de la orden de producción: el jefe de producción informa de manera verbal la producción del día y procede a entregar las hojas de trabajo a los encargados de área.

- Retiro de la materia prima a utilizar según O.P: el personal del área de acondicionado se encarga de retirar la materia prima según las especificaciones de la orden de producción.

- Acondicionado de la materia prima: el personal del área de acondicionado prepara las maquinaras para el acondicionamiento de la materia prima. El personal de acondicionado realiza el lavado y pelado de la materia prima, asimismo se realiza el picado para luego enviar la materia prima picada al área de fritado y selección.

- Fritado y selección: el personal del área de fritado realiza la recepción la materia prima 
Daniel Tejada y Giner Guadalupe

picada. Por otro lado, se preparan las cocinas para luego realizar el fritado de la materia prima. El personal de selección realiza el sazonado y selección de los productos defectuosos para después embolsarlos y pasarlos al área de envasado

- Envasado: el personal de envasado recibe los productos a granel, asimismo el supervisor del área de envasado realiza la programación de envasado. Los maquinistas preparan las máquinas para el adecuado envasado del producto final. Se realiza el entirado, encajado y rotulado por parte del personal de envasado para luego enviar las cajas de productos terminados al almacén.

- Almacenamiento: el personal del almacén realiza la recepción de los productos terminado para luego almacenarlos.

\section{Descripción del proceso de distribución}

- Recepción e ingreso de pedidos: el ejecutivo de atención al cliente recibe e ingresa al sistema ERP todos los pedidos recibidos.

- Verificación de stock: el encargado del almacén de productos terminados verifica el stock disponible para proceder con la confirmación de la fecha de atención y embalado de los productos.

- Preparación de picking y packing: el personal del área realiza el embalado del pedido de acuerdo con las indicaciones del encargado de almacén.

- Generación de documentación: el ejecutivo de Atención al Cliente genera las guías de remisión, solicita el certificado de calidad de ser requerido por el cliente. Asimismo, realiza la programación de las atenciones y asigna los vehículos para cumplir con las atenciones.
- Estiba del producto: el personal de reparto estiba los productos de acuerdo con las indicaciones de los conductores quienes siguen las indicaciones recibidas por el ejecutivo de atención al cliente y cotejan dicha información con la guía de remisión remitente.

- Distribución de la mercadería: el personal de reparto realiza la atención de la mercadería siguiendo las instrucciones recibidas por el ejecutivo de atención al cliente, si en la atención existiera un rechazo o atención parcial, los conductores son los responsables de informar al ejecutivo de atención al cliente todas las incidencias ocurridas.

- Entrega de documentos: los conductores son los responsables de la entregar toda la documentación que interviene en el proceso de distribución al ejecutivo de atención al cliente, quien recibe las incidencias y documentación para luego derivar las incidencias al área que corresponde.

- Facturación de pedido: el ejecutivo de atención al cliente realiza la facturación del pedido y notifica al cliente el comprobante de pago.

\section{Descripción de la propuesta del proceso de aprovisionamiento}

- Solicitudes de compras: el almacenero recibe las solicitudes de compras de todas las áreas.

- Evaluación de las solicitudes de compras: el responsable de almacén realiza la evaluación de las solicitudes de compras y si cumplen con los requisitos se procede a emitir y notificar el requerimiento de compra al área de administración 
- Recepción de los requerimientos de compras: el área de administración recibe los requerimientos de compras y procede a evaluar el proveedor adecuado.

- Evaluación de proveedores: el administrador solicita cotizaciones cuando se trata de una primera compra de un producto; luego recibe las cotizaciones, preselecciona al proveedor para luego evaluar y asignarlo.

- Emisión de orden de compra: el administrador emite la orden de compra de acuerdo a las indicaciones especificadas en los requerimientos de compras.

- Despacho de mercadería: proveedor realiza la entrega de mercadería de acuerdo a las especificaciones de la orden de compra.

- Recepción de mercadería: el almacenero realiza la verificación y recepción de la mercadería, asimismo corrobora que todos los documentos se hayan emitido de manera adecuada

- Ingreso a almacén: una vez verificado la mercadería, el almacenero procede a ingresar la mercadería al almacén.

- Registro del ingreso de mercadería: el almacenero realiza el registro del ingreso de mercadería en el sistema ERP.

- Entrega de documentos: el almacenero remite los documentos al área contable para su provisión y pago.

- Registro contable y provisión de pago: el analista contable realiza el registro contable de la compra y la provisión del pago.

\section{Descripción de la propuesta del proceso de producción}

- Recepción del pedido: el jefe de producción recibe la notificación del pedido.

- Generación de la orden de producción: el jefe de producción genera la orden de producción en el sistema ERP.
- Requisición de materiales: el almacenero recibe el requerimiento de materia prima a utilizarse en la producción.

- Aprobación y registro de salida de materia prima: el almacenero aprueba y registra la salida de materia prima en el sistema ERP.

- Recepción de la materia prima: el personal del área de acondicionado recibe la materia prima a utilizarse para la producción.

- Selección de la materia prima defectuosa: el personal del área de acondicionado, seleccionada los productos defectuosos y los clasifica en mermas y desmedros

- Preparación de maquinarias: el personal del área de acondicionado pone a punto las maquinarias para el acondicionamiento de la materia prima.

- Acondicionamiento de la materia prima: el personal del área de acondicionado realiza el lavado y pelados de la materia prima, rectifican y pican la metería prima, para luego enviarlas al área de fritado $y$ selección.

- Fritado y selección: el personal del área de fritado recibe la materia prima picada y prepara las cocinas para el fritado. El personal del área de selección sazona y selecciona los productos aptos para la venta. El responsable del área de selección registra las cantidades de mermas y desmedros generados en el proceso de selección. El personal de selección realiza el envasado del producto a granel. El responsable del área de selección registra la cantidad de producto a granel producido.

- Envasado: el área de envasado verifica la calidad del producto en proceso y procede a recibir la mercadería. Los maquinistas preparan las maquinas envasadoras para el correcto envasado del producto final. El 
personal del envasado realiza el encajado y rotulado del producto final.

- Almacenamiento: el almacenero recibe el producto final y registra en el sistema ERP. También realiza el mantenimiento adecuado de los productos terminados.

\section{Descripción de la propuesta del proceso de distribución}

- Recepción de pedidos: el responsable del almacén de productos terminados, reciben todos los pedidos.

- Verificación del stock: el almacenero verifica el stock disponible en el sistema ERP

- Notificación al cliente: Notifica al vendedor la conformidad del pedido.

- Emisión de la orden de salida: emite la orden de salida para autorizar el retiro de mercadería del almacén.

- Registro de la salida de mercadería: registra en el sistema ERP la salida de productos.

- Emisión del picking: emite una orden de picking para el alistamiento del pedido.

- Preparación - packing: el personal de almacén realiza el packing de acuerdo con las indicaciones del jefe almacenero.

- Recepción de los productos para despacho: el auxiliar de despacho recibe los productos embalados y listos para despacho.

- Realización del control de calidad: el auxiliar de despacho realizar el control de calidad de los productos embalados y a la vez verificar el adecuado alistamiento del pedido.

- Programación de atención: el auxiliar de despacho programa las atenciones.

- Elaboración de rutas y asignación de vehículos: el auxiliar de despacho elabora las rutas y asigna los vehículos adecuados para las atenciones afín de cumplir con la programación establecida.
- Emisión de las guías de remisión: el auxiliar de despacho emite las guías de remisión remitente y procede a entregar toda la documentación al personal de transporte.

- Estiba y distribución: el personal de reparto carga los productos al vehículo para luego cumplir con la distribución de acuerdo con lo establecido en la programación.

- Notificación de incidencias: los conductores comunican las incidencias ocurridas en el proceso de atención.

- Gestión de incidencias: el ejecutivo de atención al cliente recibe las incidencias ocurridas en el proceso de atención y procede a derivarla al departamento competente.

- Recepción y custodia de guías de remisión remitente: el ejecutivo de atención al cliente recibe y archiva los documentos que interviene en el proceso de distribución.

- Facturación de atención realizada: el ejecutivo de atención al cliente realiza la facturación de los pedidos entregados en su totalidad

\section{Propuesta.}

La propuesta de mejora esta argumentada en el análisis de evaluación de la matriz de evaluación de control interno y los flujogramas de identificación de riesgos como herramientas esenciales para el reconocimiento de las debilidades en los subprocesos de aprovisionamiento, producción y distribución que forman parte del proceso logístico y mediante el cual se presentaron una serie de propuestas tomando como referencia los cinco componentes del modelo coso.

a) Entorno de control: en relación a este componente y afín de mejorar la gestión logística, se propone una nueva estructura 
orgánica para el departamento de logística con responsabilidad definida ya que la empresa Redjos al ser una empresa del sector industrial concentra un alto volumen de operaciones de compras, abastecimiento e inventarios por lo cual le es importante que se implemente dicho departamento, estableciéndose de esta manera líneas de reporte, autoridad y responsabilidad apropiada para la consecución de los objetivos.

b) Evaluación de riesgos: respecto a este componente se propone elaborar un planeamiento estratégico en donde se encuentren definidos los objetivos que la empresa persigue a corto, mediano y largo plazo. Por otra parte, también se propone hacer uso de las siguientes herramientas para una adecuada identificación y evaluación de riesgos.

- Matriz de identificación de riesgos.

- Plan de administración de riesgos, como estrategia a la respuesta de los riesgos.

- Flujogramas de procesos para la identificación de riesgos.

c) Actividades de control: en relación con este componente se propone elaborar un manual de operaciones y funciones (MOF) para el departamento de logística afín de garantizar que el personal involucrado en el proceso logístico conozca sus responsabilidades y funciones.

Por otro lado, para disminuir los riesgos que pueden afectar el logro de objetivos del proceso logístico se propone implementar las siguientes políticas y procedimientos.

- Plan de compras

- Políticas de compras
- Procedimientos adecuados para la evaluación de proveedores.

- Procedimientos adecuados para la recepción y aprobación de requerimientos de compras.

- Procedimientos adecuados para la recepción de mercadería de compras.

- Políticas de control de inventario

- Método de evaluación de inventarios

- Política de devoluciones

- Plan maestro de producción

- Procedimientos adecuados para la gestión de pedidos

- Plan de mantenimiento preventivo maquinarias y vehículos.

d) Información y comunicación: en relación con este componente afín de lograr integrar y procesar la información de los cinco componentes se propone que todo el proceso logístico se encuentre debidamente documentado a fin de garantizar una información de calidad y oportuna para la preparación y elaboración de la información financiera, por lo tanto, la siguiente propuesta para su implementación en el sistema ERP son:

Reportes no financieros

- Reportes de evaluación de proveedores

- Reportes de mermas y desmedros

- Reporte de Requerimiento de compras pendientes de aprobación.

- Reportes de inventarios Kardex

- Reporte de seguimiento de órdenes de compras.

- Reporte de pedidos pendientes de atención

- Reportes de pedidos rechazados 
Daniel Tejada y Giner Guadalupe

e) Actividades de supervisión y monitoreo: en relación con este componente debemos de tener en cuenta que la incorporación de actividades de autocontrol en los procesos y operaciones de supervisión serán de utilidad para valorar la eficacia del control interno, por lo tanto, para el mejoramiento de los procesos logísticos se plantea una serie de indicadores de gestión logística que ayudar a medir la eficiencia de las actividades del proceso logístico, los cuales se presentan a continuación:

Tabla 1. Indicadores de gestión logística

\begin{tabular}{|c|c|c|c|}
\hline Indicadores & Objetivo & Cálculo & Proceso Logístico \\
\hline $\begin{array}{l}\text { Certificación de } \\
\text { proveedores }\end{array}$ & & $\begin{array}{l}\text { Valor }=\text { proveedores } \\
\text { certificados / total } \\
\text { proveedores. }\end{array}$ & $\begin{array}{l}\text { Aprovisionamiento } \\
\text { /compras }\end{array}$ \\
\hline $\begin{array}{c}\text { Calidad de } \\
\text { pedidos generados }\end{array}$ & & $\begin{array}{c}\text { Valor }=\text { pedidos generados } \\
\text { sin problemas / total pedidos } \\
\text { generados * } 100\end{array}$ & $\begin{array}{c}\text { Aprovisionamiento / } \\
\text { compras }\end{array}$ \\
\hline $\begin{array}{l}\text { Volumen de } \\
\text { compras }\end{array}$ & $\begin{array}{l}\text { Controlar la evolución } \\
\text { del volumen de } \\
\text { compra en relación } \\
\text { con el volumen de } \\
\text { ventas. }\end{array}$ & $\begin{aligned} \text { Valor }= & \text { Valor de compra } / \\
& \text { total ventas }\end{aligned}$ & $\begin{array}{l}\text { Aprovisionamiento } \\
\text { /compras }\end{array}$ \\
\hline $\begin{array}{l}\text { Entregas } \\
\text { perfectamente } \\
\text { recibidas }\end{array}$ & $\begin{array}{l}\text { Controlar la calidad } \\
\text { de los productos } \\
\text { recibidos y la } \\
\text { puntualidad de } \\
\text { entregas de los } \\
\text { proveedores. }\end{array}$ & $\begin{array}{l}\text { Pedidos rechazados / total } \\
\text { ordenes de compras } \\
\text { recibidas * } 100\end{array}$ & $\begin{array}{c}\text { Aprovisionamiento / } \\
\text { Almacén }\end{array}$ \\
\hline $\begin{array}{l}\text { Capacidad de } \\
\text { producción } \\
\text { utilizada }\end{array}$ & $\begin{array}{c}\text { Controlar la } \\
\text { utilización efectiva de } \\
\text { las instalaciones de la } \\
\text { empresa. }\end{array}$ & $\begin{array}{l}\text { Capacidad utilizada / } \\
\text { capacidad máxima de } \\
\text { recursos }\end{array}$ & Producción \\
\hline
\end{tabular}

Fuente: Tejada, Guadalupe 2019. 


\begin{tabular}{|c|c|c|c|}
\hline Indicadores & Objetivo & Cálculo & Proceso Logístico \\
\hline $\begin{array}{l}\text { Rendimiento de } \\
\text { máquina. }\end{array}$ & $\begin{array}{l}\text { Controlar la } \\
\text { productividad de } \\
\text { una máquina de } \\
\text { manufactura con } \\
\text { respecto a la } \\
\text { capacidad máxima } \\
\text { de utilización. }\end{array}$ & $\begin{array}{l}\text { Número de unidades } \\
\text { producidas / capacidad } \\
\text { máxima de recurso * } 100\end{array}$ & Producción \\
\hline $\begin{array}{l}\text { Rotación de } \\
\text { mercadería }\end{array}$ & $\begin{array}{l}\text { Controlar la } \\
\text { cantidad de } \\
\text { productos } \\
\text { despachados desde } \\
\text { el centro de } \\
\text { distribución. }\end{array}$ & $\begin{array}{l}\text { Ventas acumuladas/ } \\
\text { inventario promedio }\end{array}$ & Distribución / almacén \\
\hline $\begin{array}{l}\text { Duración de } \\
\text { inventario }\end{array}$ & $\begin{array}{l}\text { Controlar los días } \\
\text { de inventario } \\
\text { disponible de la } \\
\text { mercadería } \\
\text { almacenada en el } \\
\text { centro de } \\
\text { distribución. }\end{array}$ & $\begin{array}{l}\text { Inventario final / ventas } \\
\text { promedio* } 30\end{array}$ & Distribución / almacén \\
\hline $\begin{array}{l}\text { Vejez del } \\
\text { inventario }\end{array}$ & $\begin{array}{l}\text { Controlar el nivel de } \\
\text { las mercaderías no } \\
\text { disponibles para } \\
\text { despacho por } \\
\text { obsolescencias, mal } \\
\text { estado y otros. }\end{array}$ & $\begin{array}{c}\text { Unidades dañadas + } \\
\text { obsoletas + vencidas / } \\
\text { unidades disponibles de } \\
\text { inventario }\end{array}$ & Distribución / inventarios. \\
\hline $\begin{array}{l}\text { Valor económico } \\
\text { del inventario }\end{array}$ & $\begin{array}{l}\text { Medir y controlar el } \\
\text { valor del inventario } \\
\text { promedio respecto } \\
\quad \text { a las ventas. }\end{array}$ & $\begin{array}{l}\text { Costo venta del mes / valor } \\
\text { inventario físico }\end{array}$ & Distribución / almacén \\
\hline $\begin{array}{l}\text { Exactitud en } \\
\text { inventarios }\end{array}$ & $\begin{array}{l}\text { Controlar y medir la } \\
\text { exactitud de los } \\
\text { inventarios en pos } \\
\text { de mejorar la } \\
\text { confiabilidad. }\end{array}$ & $\begin{array}{l}\text { Valor diferencia / valor } \\
\text { total inventario *100 }\end{array}$ & $\begin{array}{c}\text { Distribución } \\
\text { Aprovisionamiento / } \\
\text { almacén }\end{array}$ \\
\hline
\end{tabular}


Daniel Tejada y Giner Guadalupe

\begin{tabular}{|c|c|c|c|}
\hline Indicadores & Objetivo & Cálculo & Proceso Logístico \\
\hline $\begin{array}{l}\text { Costo de unidad } \\
\text { almacenada }\end{array}$ & $\begin{array}{c}\text { Controlar el valor } \\
\text { unitario del costo } \\
\text { de almacenamiento } \\
\text { propio. }\end{array}$ & $\begin{array}{l}\text { Costo almacenamiento / } \\
\text { N. }{ }^{\circ} \text { de unidades } \\
\text { almacenadas }\end{array}$ & Distribución/ almacén \\
\hline $\begin{array}{l}\text { Nivel de } \\
\text { cumplimiento de } \\
\text { despachos }\end{array}$ & $\begin{array}{l}\text { Controlar la eficacia } \\
\text { de los despachos } \\
\text { efectuados por el } \\
\text { centro de } \\
\text { distribución }\end{array}$ & $\begin{array}{c}\mathrm{N} .^{\circ} \text { de despachos } \\
\text { cumplidos a tiempo / N. } \\
\text { total de despachos } \\
\text { requeridos }\end{array}$ & Distribución / almacén \\
\hline Entregas perfectas & $\begin{array}{c}\text { Conocer la } \\
\text { eficiencia de los } \\
\text { despachos } \\
\text { efectuados por la } \\
\text { empresa teniendo } \\
\text { en cuenta las } \\
\text { características de } \\
\text { los completos a } \\
\text { tiempo, con } \\
\text { documentación y } \\
\text { sin daño de } \\
\text { mercadería. }\end{array}$ & $\begin{array}{c}\text { Pedidos entregados } \\
\text { perfecto / total de pedidos } \\
\text { entregados. }\end{array}$ & $\begin{array}{c}\text { Distribución/ servicio al } \\
\text { cliente }\end{array}$ \\
\hline
\end{tabular}

\section{CONCLUSIONES}

De acuerdo con la información presentada en esta investigación, relacionada con la evaluación del control interno para mejorar la gestión logística en la empresa Redjos S.A.C, se presenta lo siguiente.

En relación con el primer objetivo específico, se evidenció la ausencia del entorno de control en los procesos logísticos debido a que la entidad no cuenta con una estructura orgánica que defina las responsabilidad, autoridad y líneas de reportes apropiados para la consecución de los objetivos, esto se refleja en el $47 \%$ de eficacia alcanzado en la evaluación.

Por otro lado, en el segundo objetivo específico, se encontró que la evaluación de riesgos es no eficaz en un $83.33 \%$, a su vez mediante los flujogramas de identificación de riesgos se sabe que los procesos logísticos carecen de un plan adecuado para la gestión y mitigación de riesgos, por lo que es importante que esto se mejore elaborando para ello un planeamiento estratégico para el departamento logístico.

Respecto al tercer objetivo específico, se evidenció las actividades de control del proceso logístico mediante la matriz de evaluación del control interno, en donde se observó que la entidad solo aplica actividades de control con eficacia en un $33.33 \%$, por lo cual carece de controles adecuados como políticas y procedimientos que ayuden a minimizar los riesgos que puedan afectar el logro de los objetivos, por ello es importante que se preste mayor atención a la formalización de políticas y 
procedimientos que se acerquen a la realidad de la empresa y de esta forma ayuden a mitigar los riesgos.

Respecto al cuarto objetivo específico, se observó que la información y comunicación en los procesos logísticos carece de procedimientos adecuados y formalizados, debido a que la información generada en todo el proceso logístico es importante para apoyar al buen funcionamiento del control interno y por consiguiente lograr una mejor gestión logística. Se encontró que este se viene aplicando eficazmente en un $11.11 \%$, necesitando así continuar con el trabajo de unificar las relaciones entre las diferentes áreas

Respecto al quinto objetivo específico, se evidenció en la descripción de las actividades de los procesos logísticos que estos no cuentan con actividades de autocontrol adecuados e incorporados en sus procesos por lo cual es importante que se implementen controles eficientes que contribuyan a supervisar y evaluar los controles internos del proceso logístico. Así también, se observó que la empresa alcanzó a aplicar eficazmente en un 50\% las actividades de monitoreo y supervisión.

En relación con el sexto objetivo específico, del análisis de la evaluación del control interno, se propuso un nuevo flujograma del proceso logístico a fin de mejorar la gestión logística en la empresa Redjos S.A.C.

Finalmente, respecto al objetivo general se concluye que la empresa Redjos S.A.C., viene aplicando un control interno de forma empírica lo cual ante el constante crecimiento en el volumen de operaciones, actividades, recursos, empleados y exigencias de un mercado competitivo generará dificultades para adaptar su estructura orgánica a entornos económicos competitivos y en consecuencia ocasionará pérdidas económicas para la entidad; por ello consideramos importante al control interno basado en el modelo COSO como una herramienta que provee de información confiable para una conducción ordenada y eficiente de las actividades; en consecuencia al contar con un sistema de control interno eficiente, las actividades logísticas también se encontrarán debidamente integradas y eficientes, generando de esta manera una ventaja competitiva para la empresa y una fácil adaptación a nuevos mercados y exigencias de los clientes.

\section{REFERENCIAS}

Contraloría General de la República. (2014). Marco conceptual del control interno. Deutsche Gesellschaft Für Internationale Zusammenarbeit (GIZ) GmbH Programa Buen Gobierno y Reforma Del Estado, 62. Retrieved from http://www.minam.gob.pe/comite/wpcontent/uploads/sites/67/2014/10/Marcoconceptual-del-Cl.pdf

Cuellar, G. (2009). Informe ejecutivo COSO. Universidad Del Cusco, 116. Retrieved from https://preparatorioauditoria.wikispaces.co $\mathrm{m} /$ file/view/Informe+Ejecutivo+COSO.pdf

García, A. (2010). Gestión logística integral. (E. Ediciones, Ed.) (Segunda Ed).

Hernández, C., Fernández, C., \& Baptista, P. (2014). Metodología de la investigación (Sexta Edic). México: Mc Graw Hill / Interamericana Editores S.A.

Kirby, C., \& Brosa, N. (2011). La logística como factor de competitividad de las Pymes en las Américas, 39. Retrieved from https://publications.iadb.org/publications/s panish/document/La-log\%C3\%ADsticacomo-factor-de-competitividad-de-lasPymes-en-las-Am\%C3\%A9ricas.pdf

Resumen ejecutivo COSO II. (n.d.).

Rivas, G. (2011). Modelos contemporáneos de 
control interno. Fundamentos teóricos. Observatorio Laboral Revista Venezolana, 4(8), 115-136. https://doi.org/Observatorio Laboral Revista Venezolana Vol. 4, No 8, julio-diciembre, 2011: 115-136

Servin, L. (2018). ¿Porque es importante el control interno en las empresas? Retrieved from

https://www2.deloitte.com/py/es/pages/au dit/articles/opinion-control-internoempresas.html\#

Ubillús, J., Tamayo, C., \& Zambrano, M. (2016). El Control Interno como Herramienta Eficiente en las Pymes de la ciudad de
Portoviejo, 7, 6-15. Retrieved from http://186.46.160.238/revistas/index.php/ec asinergia/article/viewFile/306/219 Control. Contaduría Universidad de Antioquia, 69, 139-154.

Villa, C., \& Vargas, D. (2016). Marco integrado de control interno - COSO III.

Young, N. (2013). El control interno es necesario para preservar el negocio. Diario Gestion. Retrieved from https://gestion.pe/tendencias/management -empleo/pwc-control-interno-necesariopreservar-negocio-46435 